\title{
Vergleich von fünf kombinierten anti-HIV-1/2-ELISA mit zwei verschiedenen Serokonversionspanels
}

\section{Comparison of five anti-HIV-1/2-ELISA using two different Seroconversion Panels}

K. Koerner, Dagmar Kilian

Blutspendezentrale Ulm des DRK-Blutspendedienstes Baden-Württemberg

\section{Zusammenfassung:}

Die diagnostische Sensitivität der z. Z. in der Bundesrepublik Deutschland vom Paul-Ehrlich-Institut zum Verkauf zugelassenen Tests zur kombinierten Testung auf anti-HIV-1/2 in Serum oder Plasma wurde mit zwei antiHIV-1-Serokonversionspanels von Plasmapheresespendern untersucht. Die Tests unterscheiden sich in der Erkennung der Serokonversion bis zu 21 Tagen in diesen untersuchten Serokonversionspanels.

Schlüsselwörter: .

anti-HIV-1/2-ELISA - Sensitivität - Serokonversion

\section{Summary:}

The sensitivity of the five Paul-Ehrlich-Institut-licensed anti-HIV-1/2-ELISA for screening of serum or blood plasma in the Federal Republik of Germany was evaluated by using two seroconversion panels of anti HIV-1.

The combined anti-HIV-1/2-ELISA tests show differences up to 21 days in the sensitivity to detect anti-HIV-1 antibodies.

Keywords:

anti-HIV-1/2-ELISA - sensitivity - seroconversion

\section{Einleitung}

Seit der Einführung kommerzieller anti-HIV-1-ELISA im Jahre 1985 zum Screening aller Blutspender wurden die verschiedenen Testverfahren bezugnehmend auf die diagnostische Sensitivität und Spezifität wesentlich verbessert $(1,2)$. Mit dem Auftreten von HIV-2-Infektionen auch in der Bundesrepublik Deutschland wurde im Februar 1989 von der Regierung in Oberbayern gefordert, daß zu den bisherigen Maßnahmen zur Vermeidung der Übertragung von HIV-2 durch Blut- und Blutbestandteilkonserven auch ein anti-HIV-2-Test mit einzubeziehen ist. Die Einführung des Tests auf HIV-2-Antikörper anläßlich jeder Blutspende wird nach bisher bekannt gewordenem Stufenplanverfahren vom Bundesgesundheitsamt bis zum 1. Januar 1990 zur Auflage gemacht. Die Forderung für einen kombinierten Test anti-HIV-1 und anti-HIV-2 war, daß die diagnostische Sensitivität und Spezifität nicht schlechter als bei den bisherigen anti-HIV-1-Tests sein sollte (3). Fünf verschiedene, kombinierte anti-HIV-1/2-ELISA wurden bisher vom Paul-Ehrlich-Institut zur kombinierten Testung von Serum oder Plasma zugelassen. Die Seren zweier verschiedener Serokonversionspanels haben wir auf antiHIV-1 mit diesen Tests untersucht.

\section{Material und Methoden}

Alle Tests wurden nach den Angaben der Hersteller durchgeführt und die testspezifischen Qualitätskontrollen beachtet. Alle Seren wurden in zwei unabhängigen Testläufen doppelt untersucht. Als Serokonversionspanel wurde Panel A mit 9 Proben und Panel $\mathrm{C}$ mit 18 Proben der Fa. Boston Biomedica, Inc., USA, verwendet. Die Proben wurden tiefgefroren aus den USA angeliefert; jedes Panel besteht aus einer Serie von Plasmaproben eines Probanden. Von diesem anti-HIV-1-positiven Probanden standen über einen längeren Zeitraum vor, während und nach der Serokonversion Plasmaproben zur Verfügung.

Folgende Tests wurden untersucht:

(a) Abbott Recombinant HIV-1/2-EIA

Ch.-Nr. 31767

(HIV-1: CORE u. ENV-Antigene; HIV-2: ENV-Antigen) rekombinante Antigene

(b) Behring Enzygnost-Anti-HIV-1 +2

Ch.-Nr. 20243

(HIV-1: $2 \times$ gp 41, $1 \times$ gp 120; HIV-2: $1 \times$ gp 36)

Peptide

(c) Biochrom KG HIV-1/-2-ELISA

Ch.-Nr. 012589

(HIV-1: gp 41. gp 120; HIV-2: gp 36)

Peptide

(d) Pasteur Diagnostika Rapid ELAVIA MIXT

Ch.-Nr. 9C $412 \mathrm{Y}$

(HIV-1, HIV-2: gereinigtes, inaktiviertes Virusantigen)

(e) Wellcome Wellcozyme HIV $1+2$

Ch.-Nr. K 800010

(HIV-1: rekombinantes ENV und CORE-Protein; HIV-2: ENV-Peptid) 


\section{BIOTECH/Du Pont HIV-1 Western blot IgG} Ch.-Nr. 9244

Die Bewertung wurde nach folgenden Kriterien durchgeführt:

negativ: keine virusspezifische Proteinbande

positiv: gp 41 alleine, p 24 und gp 120, oder gp 41 und eine andere virusspezifische Bande.

fraglich: p24 alleine, eine andere virusspezifische Bande oder die Kombination mehrerer Banden in allen anderen Bereichen.

\section{Ergebnisse und Diskussion}

In den Tabellen 1 und 2 sind die Testergebnisse aufgelistet. Im Testpanel A reagierten die Tests $a, b$ und e zum gleichen Zeitpunkt positiv, während die Tests $c$ und $d$ noch keine Reaktion zeigten. Erst bei der darauffolgenden Probenahme, die 3 Wochen danach erfolgte, konnte auch mit Test $c$ und $d$ eine positive Reaktion nachgewiesen werden. Im Testpanel $C$ zeigen die Tests a und $b$ die. selbe diagnostische Sensitivität, während Test $d$ und $e$ zwei bzw. sieben Tage später einen positiven Befund auf-

Tab. 1: Anti-HIV-1-Antikörper bei Serokonversion. Serokonversionspanel A: Boston Biomedica, Inc., USA. Anti-HIV-1/2-ELISA, vom Paul-Ehrlich-Institut zugelassen.

\begin{tabular}{|c|c|c|c|c|c|c|c|}
\hline \multirow[t]{2}{*}{ Probe } & \multirow[t]{2}{*}{ Spendedatum } & \multicolumn{5}{|c|}{ Anti-HIV-1/2-ELISA (s/co)* } & \multirow{2}{*}{$\begin{array}{c}\text { Anti-HIV-1 } \\
\text { Western blot } \\
\text { Dupont }\end{array}$} \\
\hline & & $\mathbf{a}$ & b & c & d & e & \\
\hline $1 A$ & 040581 & $0,54(-)$ & $0,18(-)$ & $0,37(-)$ & $0,29(-)$ & $0,36(-1$ & $+1-* *$ \\
\hline $2 A$ & 080781 & $0,38(-)$ & $0,07(-)$ & $0,61(-)$ & $0,48(-)$ & $0,34(-)$ & $+1-* *$ \\
\hline 3A & 290781 & $3,42(+)$ & $4,361+1$ & $0,38(-1$ & $0,681-1$ & $4,50(+)$ & + \\
\hline $4 A$ & 190881 & $11,6(+)$ & $8,951+1$ & $2,44(+)$ & $5,52(+)$ & $5,30(+)$ & + \\
\hline $5 A$ & 020981 & $13,9(+)$ & $8,95(+)$ & $8,05(+)$ & $5,95(+)$ & $6,40(+)$ & + \\
\hline $6 A$ & 090981 & $11,1(+)$ & $8,95(+1$ & $8,88(+1$ & $5,95(+)$ & $6,20(+)$ & n.d. \\
\hline $7 A$ & 160981 & $15,6(+)$ & $8,95(+)$ & $8,88(+)$ & $5,95(+)$ & $7,40(+)$ & n.d. \\
\hline $8 \mathrm{~A}$ & 230981 & $15,6(+)$ & $8,95(+)$ & $8,88(+)$ & $5,95(+)$ & $7,13(+)$ & n. d. \\
\hline $9 A$ & 141081 & $15,6(+)$ & $8,95(+)$ & $8,88(+)$ & $5,95(+)$ & $7,53(+)$ & n. d. \\
\hline
\end{tabular}

- $s / c 0=$ Quotient aus Extinktion der Probe zu cut off.

Beurteilung:

Quotient $\geq 1.0=$ anti-HIV1 positiv $(t)$

$\geq 0.9-\leq 1.0=$ anti-HIV1 fraglich $(+1-)$, Grauzone $\leq 0.9=$ anti-HIV1 negativ.

Die Werte sind Mittelwerte aus zwei getrennten Testansätzen

*" nur p 24, n. d. = nicht durchgeführt.

Tab. 2: Anti-HIV-1-Antikörper bei Serokonversion. Serokonversionspanel C: Boston Biomedica, Inc., USA. Anti-HIV-1/2-ELISA, vom Paul-Ehrlich-Institut zugelassen.

\begin{tabular}{|c|c|c|c|c|c|c|c|}
\hline \multirow[t]{2}{*}{ Probe } & \multirow[t]{2}{*}{ Spendedatum } & \multicolumn{5}{|c|}{ Anti-HIV-1/2-ELISA (s/co)* } & \multirow{2}{*}{$\begin{array}{l}\text { Anti-HIV-1 } \\
\text { Western blot } \\
\text { Dupont }\end{array}$} \\
\hline & & $\mathbf{a}$ & $\mathbf{b}$ & c & d & $\mathbf{e}$ & \\
\hline $1 \mathrm{C}$ & 160785 & $0,48(-)$ & $0,06(-)$ & $0,13(-)$ & $0,22(-)$ & $0,30(-)$ & - \\
\hline $2 \mathrm{C}$ & 230785 & $0,47(-)$ & $0,11(-)$ & $0,14(-)$ & $0,16(-)$ & $0,42(-)$ & - \\
\hline $3 C$ & 250785 & $0,86(-)$ & $0,84(-)$ & $0,13(-)$ & $0,36(-)$ & $0,58(-)$ & $+1-* *$ \\
\hline $4 C$ & 300785 & $2,32(+)$ & $6,71(+)$ & $0,34(-)$ & $0,63(-)$ & $0,61(-)$ & + \\
\hline $5 C$ & 010885 & $2,11(+)$ & $8,79(+)$ & $0,63(-)$ & $1,00(+/-)$ & $0,75(-)$ & + \\
\hline $6 C$ & 060885 & $4,18(+)$ & $8,95(+)$ & $0,93(+1-)$ & $2,02(+)$ & $1,63(+)$ & n. d. \\
\hline $7 C$ & 080885 & $4,70(+)$ & $8,95(+)$ & $0,97(+1-)$ & $2,35(+)$ & $2,41(+)$ & n. d. \\
\hline $8 C$ & 130885 & $6,44(+)$ & $8,95(t)$ & $0,95(+/-)$ & $3,72(+)$ & $3,16(+)$ & n. d. \\
\hline $9 \mathrm{C}$ & 150885 & $7,56(+)$ & $8,95(+)$ & $1,36(+)$ & $3,79(+)$ & $4,16(+)$ & n. d. \\
\hline $10 \mathrm{C}$ & 200885 & $9,50(+)$ & $8,95(+)$ & $2,53(+)$ & $5,95(+)$ & $5,84(+)$ & n. d. \\
\hline $11 \mathrm{C}$ & 270885 & $13,40(+)$ & $8,95(+)$ & $4,20(+)$ & $5,95(+)$ & $6,43(+)$ & n. d. \\
\hline $12 \mathrm{C}$ & 290885 & $10,10(+)$ & $8,95(+)$ & $3,50(+)$ & $5,95(+)$ & $6,38(+)$ & n. d. \\
\hline $13 C$ & 100985 & $13,80(+)$ & $8,95(+)$ & $4,95(+)$ & $5,95(+)$ & $6,68(+)$ & n. d. \\
\hline $14 C$ & 120985 & $8,45(+)$ & $8,95(+)$ & $4,68(+)$ & $5,995(+)$ & $6,58(+)$ & n. d. \\
\hline $15 \mathrm{C}$ & 170985 & $14,60(+)$ & $8,95(+)$ & $5,67(+)$ & $5,95(+)$ & $6,58(+)$ & n. d. \\
\hline $16 \mathrm{C}$ & 190985 & $12,80(+)$ & $8,95(+)$ & $5,48(+)$ & $5,95(+)$ & $6,93(+)$ & n. d. \\
\hline $17 C$ & 240985 & $13,90(+)$ & $8,95(+)$ & $4,75(+)$ & $5,95(+)$ & $7,48(+)$ & n. d. \\
\hline $18 C$ & 260985 & $15,60(+)$ & $8,95(+)$ & $3,90(+)$ & $5,95(+)$ & $7,53(+)$ & n. d. \\
\hline
\end{tabular}

* $\mathrm{s} / \mathrm{co}=$ Quotient aus Extinktion der Probe zu cut off.

Beurteilung:

Quotient $\geq 1.0=$ anti-HIV1 positiv $(t)$

$\geq 0.9-\leq 1.0=$ anti-HIV1 fraglich $(+/-)$, Grauzone $\leq 0.9=$ anti-HIV1 negativ.

Die Werte sind Mittelwerte aus zwei getrennten Testansätzen.

** nur p 24, n. d. = nicht durchgeführt. 
weisen. Die Ergebnisse bei Test $\mathrm{c}$ liegen bis zu 14 Tagen später noch im Grauzonenbereich (Tabelle 2), während alle anderen ELISA eindeutige positive Ergebnisse aufweisen. Der anti-HIV-1 Western blot zeigte bei beiden Panels jeweils eine oder zwei Serumproben früher ein fragliches Ergebnis mit einer $p$ 24-Bande des core-Proteins, bevor ein anti-HIV-1/2 ELISA positiv war. Der anti-HIV-1 Western blot zeigt somit höhere diagnostische Sensitivität als die kombinierten anti-HIV-1/2-ELISA. Die diagnostische Sensitivität von anti-HIV-2 kann aufgrund dieser Untersuchung nicht beurteilt werden.

In einer früheren (Mitte 1987) vergleichenden Studie wurde ebenfalls die diagnostische Sensitivität der Serokonversionspanels $A$ und $C$ mit verschiedenen anti-HIV-1-ELISA untersucht. Zwischen den acht anti-HIV-1Tests, die von der Food and Drug Administration (FDA) zugelassen waren, wurden ebenfalls unterschiedliche $\mathrm{Er}$ gebnisse festgestellt (4).

In anderen Studien, die verschiedene kommerzielle Tests zum Nachweis von anti-HIV-1 verglichen, konnten auch Unterschiede in der diagnostischen Sensitivität und Spezifität festgestellt werden $(1,5)$, wobei für die Bestimmung der Sensitivität häufig Titrationsexperimente durchgeführt wurden $(6,7,8)$.

Ein Ringversuch der Blutspendedienste und Laboratorien, bei dem die Sensitivität und Spezifität mit 13 kommerziellen ELISAs zum Nachweis von anti-HIV-1-Antikörpern bestimmt wurde, zeigte, daß die Tests sehr verläßlich sind mit einer durchschnittlichen diagnostischen Sensitivität und Spezifität von $99,5 \%$ (9). Jedoch wurden HIV-2-Antikörper-positive Seren mit den kommerziellen anti-HIV-1ELISA mit unterschiedlicher Häufigkeit als positiv erkannt $(9,10)$. In kombinierten Tests zum Nachweis von anti-HIV-1 und 2 zeigte sich jedoch eine Empfindlichkeit von $>99 \%$ für anti-HIV-2 (11, 12, 13). Die Tests zum Nachweis von anti-HIV-Antikörpern werden ständig verbessert. Es ist daher wichtig, die Qualität dieser Tests laufend zu vergleichen (9).
Schrifttum:

1. OLDHAM, L. J., MOULSDALE, H. J., MORTIMER, P. P., TEDDER, R. S., MORGAN-CAPNER, P.: How sensitive are the commercial assays for anti-HTLVIII/LAV? J. Med. Virology 21, 75-79 (1987).

2. HESS, G, ROSSOL, S, WEBER, K, C. MEYER ZUM BÜSCHENFELDE, K. H.: Diagnose der HIV-1-Infektion: Vergleich von fünf kommerziellen Enzymimmunoassays Vergleich von fünf kommerziellen Enzymimmunoassays
zum Nachwois von anti-HIV-1. GIT Labor-Medizin 6, 361 (1988).

3. HABERMEHL, K.-O.: Neue Aspekte der Virusdiagnostik. 2. AlDS-Kongreß, S. 221, Berlin 1989.

4. SCHUMACHER, R. T., GARRETT, P. E., TEGTMEIER, G. E., THOMAS, D.: Comparative detection of anti-HIV in early HIV seroconversion. J. Clin. Immunol. 11,130 (1988).

5. GURTLER, L. G., EBERLE, J., LORBER, B., DEINHARDT, F.: Sensitivity and specifity of commercial ELISA kits for screening anti-LAV/HTLVIII. J. Virol. Methods 15, 11- 23 (1987).

6. STUTE, R.: Comparison in sensitivity of $10 \mathrm{HIV}$ antibody detection tests by serial dilutions of Western blotconfirmed samples. J. Virol. Methods 20,269-273 (1988).

7. KÜHNL, P.: HTLVIII/LAV-Antikörper-Tests: Ringversuch der Deutschen Blutspendedienste. AlDS-Forschung (AIFO) 10, 525-535 (1986).

8. ABB, J.: Determination of antibodies against LAV/ HTLVIII: comparative evaluation of four different commercial test kits. AIDS research 2, 93-97 (1986).

9. ENZENSBERGER, R., HOHN, S., KAUK, U., DOERR, H W. Sensitivity and specifity of HIV antibody tests: evaluation of a proficiency test performed by German laboratories. AIDS-Forschung 11, 622-628 (1988).

10. DENIS, F., LEONARD, G., SANGARE, A., GERSHY-DAMET, G., REY, J. L., SORO, B., SCHMIDT, D., MOUNIER, M., VERDIER, M., BAILLON, A., BARIN, F.: Comparison of 10 enzyme immunoassays for the detection of antibody to human immunodeficiency virus Type 2 in West African Sera. J. of Clin. Microbiol. 26, 1000-1004 (1988).

11. KRUPKA, U., BURST, S.: Combined screening antiHIV-1 and anti-HIV-2 with a 3rd generation assay. 2 . AIDS-Kongreß, S. 367, Berlin 1989.

12. AYRES, L., AVILEZ, F., DEINHARDT, F., GÜRTLER, L. DENIS, F., RANGER, S., GROB, P., JOLLER-JEMELKA, H., SEIDL, S., SONDAG, D., ANDRE, A., HAMPL, H. SCHOEN, R., TROONEN, H.: Multizentrische Evaluierung eines neuen EIA für die gleichzeitige Bestimmung von HIV-1- und/oder HIV-2-Antikörpern. 2. AIDS-Kongreß, S. 368, Berlin 1989.

13. SEIDL, S., FLACKE, H., SAARVEDRA, C.: Ein Screeningtest für die Erfassung von HIV-1- und HIV-2-Antikörpern in Blutspenderseren. 2. AIDS-Kongreß, S. 371, Berlin 1989 .

Anschrift der Verfasser:

Dr. Klaus Koerner

DRK-Blutspendezentrale UIm

Helmholtzstraße 10

7900 Ulm

Dagmar Kilian

DRK-Blutspendezentrale UIm

Helmholtzstraße 10

7900 UIm flasteur DAGNOSTIKA

Kallestad

\section{Screening}

HIV 1 / HIV 2

2 VIREN

1 TEST

RAPID’ ELAVIA ${ }^{\circledR}$ MIXT

ELISA zum gleichzeitigen Nachweis von HIV-1- und HIV-2-Antikörpern

Sicherheit mit Referenz in der HIV-Diagnostik

ELAVIA ${ }^{\circledR}$ Ac-Ab-Ak I ELAVIA ${ }^{\circledR}$ Ac-Ab-Ak II

Einzigartiges Testprinzip

Höchste Spezifität mittels probenbezogener Negativkontrolle im ELISA.

PASTEUR

DIAGNOSTIKA GMBH

HABSBURGERSTR. 4

D-7800 FREIBURG

TEL. NR. (0761) 5 1009-0

FAX-NR. (0761) 5 1009-99 